\title{
Steering Siglec-Sialic Acid Interactions on Living Cells using Bioorthogonal Chemistry
}

\author{
Christian Büll $^{+}$Torben Heise ${ }^{+}$, Niek van Hilten, Johan F. A. Pijnenborg, \\ Victor R. L. J. Bloemendal, Lotte Gerrits, Esther D. Kers-Rebel, Tina Ritschel, \\ Martijn H. den Brok, Gosse J. Adema ${ }^{\dagger}$ * and Thomas J. Boltje ${ }^{\dagger *}$
}

\begin{abstract}
Sialic acid sugars that terminate cell-surface glycans form the ligands for the sialic acid binding immunoglobulinlike lectin (Siglec) family, which are immunomodulatory receptors expressed by immune cells. Interactions between sialic acid and Siglecs regulate the immune system, and aberrations contribute to pathologies like autoimmunity and cancer. Sialic acid/Siglec interactions between living cells are difficult to study owing to a lack of specific tools. Here, we report a glycoengineering approach to remodel the sialic acids of living cells and their binding to Siglecs. Using bioorthogonal chemistry, a library of cells with more than sixty different sialic acid modifications was generated that showed dramatically increased binding toward the different Siglec family members. Rational design reduced cross-reactivity and led to the discovery of three selective Siglec-5/14 ligands. Furthermore, glycoengineered cells carrying sialic acid ligands for Siglec-3 dampened the activation of Siglec- $3^{+}$monocytic cells through the $N F-\kappa B$ and IRF pathways.
\end{abstract}

$T_{\text {he immune system defends the host from pathogens and }}$ cancer yet must discriminate between self and non-self to avoid damage to healthy tissue. An important differentiator in this respect is the presence or absence of sialic acid sugars on the cell surface. ${ }^{[1]}$ Sialic acids are found on the terminus of

[*] T. Heise, ${ }^{[+]}$J. F. A. Pijnenborg, V. R. L. J. Bloemendal, L. Gerrits, Dr. T. J. Boltje

Institute for Molecules and Materials

Heyendaalseweg 135, 6525 AJ, Nijmegen (The Netherlands)

E-mail: t.boltje@science.ru.nl

C. Büll, ${ }^{[+]}$E. D. Kers-Rebel, Dr. M. H. den Brok, Prof. Dr. G. J. Adema

Department of Radiation Oncology

Radiotherapy \& Oncolmmunology Laboratory

Radboud University Medical Center

Geert Grooteplein Zuid 32, 6525 GA, Nijmegen (The Netherlands)

E-mail: G.Adema@radboudumc.nl

N. van Hilten, Dr. T. Ritschel

Computational Discovery and Design Group

Centre for Molecular and Biomolecular Informatics

Radboud University Medical Center

Geert Grooteplein 26-28, 6525 GA, Nijmegen (The Netherlands)

Dr. M. H. den Brok

Department of Anesthesiology, Pain and Palliative Medicine

Radboud University Medical Center

Geert Grooteplein 10, 6525 GA, Nijmegen (The Netherlands)

$\left[{ }^{+}\right]$These authors contributed equally to this work.

['] These authors contributed equally to this work.

(2) Supporting information and the ORCID identification number(s) for

(DD the author(s) of this article can be found under:

http://dx.doi.org/10.1002/anie.201612193. cell-surface glycan chains and regulate the immune system by interacting with the sialic acid binding immunoglobulin-like lectin (Siglec) family. ${ }^{[2]}$ In humans, 14 Siglecs (Siglecs-1-11 and Siglecs-14-16) are expressed on immune cells. Siglecs contain an extracellular sialic acid binding domain and most Siglecs contain an intracellular immunoreceptor tyrosinebased inhibitory motif (ITIM; Scheme 1). ${ }^{[3]}$ Alternatively, Siglecs-14-16 can recruit an immunoreceptor tyrosine-based activation motif (ITAM). Siglecs can bind to sialic acids present on the same cell (cis binding) or sialic acids present on an adjacent cell (trans binding), and in general, these interactions dampen immune-cell activation. ${ }^{[2,3-5]}$ Aberrations in sialic acid and Siglec expression have been associated with autoimmunity, inflammatory diseases, neurodegeneration, and cancer. ${ }^{[4,6]}$ Although the importance of the sialic acid/Siglec interaction in immune regulation is well documented, its role in physiological and pathological processes is still poorly understood. ${ }^{[4]}$ To elucidate the function of sialic acid/Siglec interactions, tools are needed to specifically target Siglecs in a cellular context. This is difficult since sialic acids bind Siglecs in a monovalent manner with low affinity (0.1$3 \mathrm{~mm}) .{ }^{[2 \mathrm{cc}}$ Chemical modification of sialic acids can augment Siglec binding affinity and selectivity, and such modifications can be introduced through copper-catalyzed azide-alkyne cycloaddition (CuAAC).$^{[7,8]}$ However, these approaches are often not applicable to cell-based assays and lack multivalent presentation in a natural context, which is a crucial determinant in Siglec binding. ${ }^{[9]}$ Recently, sialoglycan-decorated polymers have been developed that can be integrated into the cell surface to bind Siglec-7. ${ }^{[10]}$ However, the use of unnatural polymers does not provide a natural context and is labor-intensive.

Therefore, we investigated the possibility of using bioorthogonal chemistry to modify sialic acids on living cells to enhance their binding to Siglecs whilst maintaining their natural multivalent presentation on the cell surface (Scheme 1). To this end, alkyne- or azide-modified sialic acids were metabolically incorporated into living cells and reacted with over sixty different azides/alkynes using CuAAC. ${ }^{[11]}$ The obtained library of glycoengineered cells was screened for binding to the human Siglec family and this led to the identification of modifications that dramatically increase Siglec binding (>100 fold). Using structure-guided design, sialic acid modifications highly selective for Siglec-5 and its homologue Siglec-14 were developed. Finally, functional analysis showed that the glycoengineered cells programmed to bind Siglec-3 are able to dampen the activation of Siglec- $3^{+}$immune cells. 


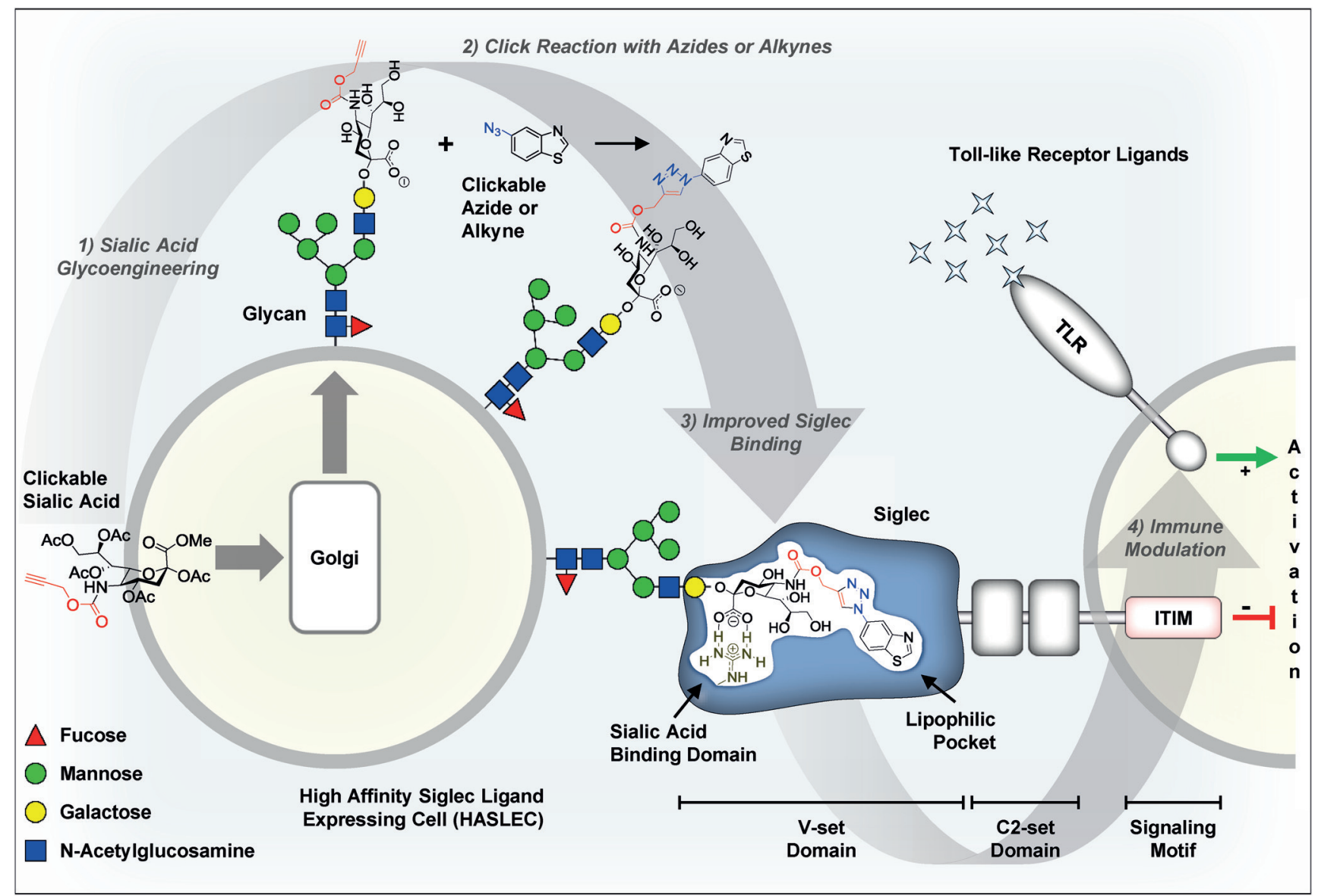

Scheme 1. Sialic acid glycoengineering on living cells to modulate Siglec-mediated immune responses.

Glycoengineering was achieved by culturing Jurkat cells in the presence of $\mathrm{Ac}_{5} \mathrm{NeuNPoc}$ or $\mathrm{Ac}_{5} \mathrm{NeuNAz}$, which results in the expression of alkyne and azide sialoglycans on the cell surface (Figure S1a; $\mathrm{Ac}_{5} \mathrm{Neu}=\mathrm{N}$-acetylneuraminic acid,

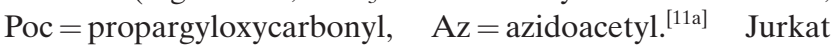
cells were used because they express $\alpha 2,3$ - and $\alpha 2,6$-sialic acids but do not express Siglecs thereby circumventing cis binding (Figures S2, S3 in the Supporting Information). To confirm the efficient incorporation of alkyne- and azidemodified sialic acids, the cells were reacted with azide- or alkyne-conjugated biotin (azide/alkyne-biotin) using $\mathrm{CuAAC}$ in the presence of histidine to minimize cytotoxicity. ${ }^{[12]}$ Biotintagged surface glycans were detected with streptavidinconjugated phycoerythrin (streptavidin-PE) by flow cytometry. As expected, high fluorescence was detected for $\mathrm{Ac}_{5} \mathrm{NeuNPoc}$ and $\mathrm{Ac}_{5} \mathrm{NeuNAz}$ but not for the control sialic acid $\mathrm{Ac}_{5} \mathrm{NeuNAc}$ (Figure S1b). Next, cells carrying alkyne- or azide-modified sialoglycans were reacted with 49 different azides (L1-L49) and 15 different alkynes (L50-L64; Figures $\mathrm{S} 4, \mathrm{~S} 5)$, respectively. To determine the reaction efficiency, a second reaction was performed with azide/alkyne-biotin to detect remaining alkynes/azides. All azides and alkynes tested showed reaction efficiencies of more than $78 \%$ (Figure 1). L1-L64 were selected on the basis of known structures and in silico docking experiments (see below). ${ }^{[8 a, 13]}$

\section{Click Efficiency}

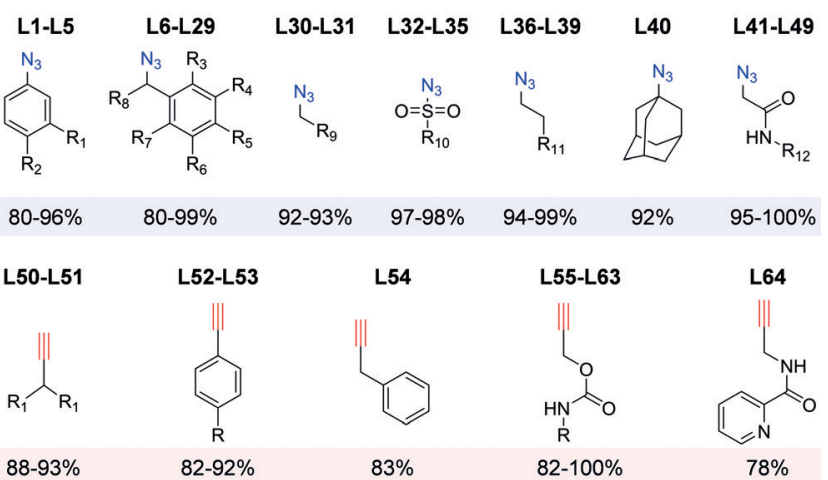

Figure 1. Generation of a sialic acid modified cell library using click chemistry.

To maximize the exploration of chemical space neighboring the sialic acid binding domain, aromatic, aliphatic, and charged azides or alkynes were synthesized. In addition, functional groups that can engage in hydrogen bonding were included (Figures S4, S5). Having demonstrated that L1-L64 react efficiently with the clickable sialic acids, the binding of available recombinant human Siglecs in the form of Silglec$\mathrm{Fc}$ chimeras $(\mathrm{Fc}=$ fragment crystallizable region of an anti- 

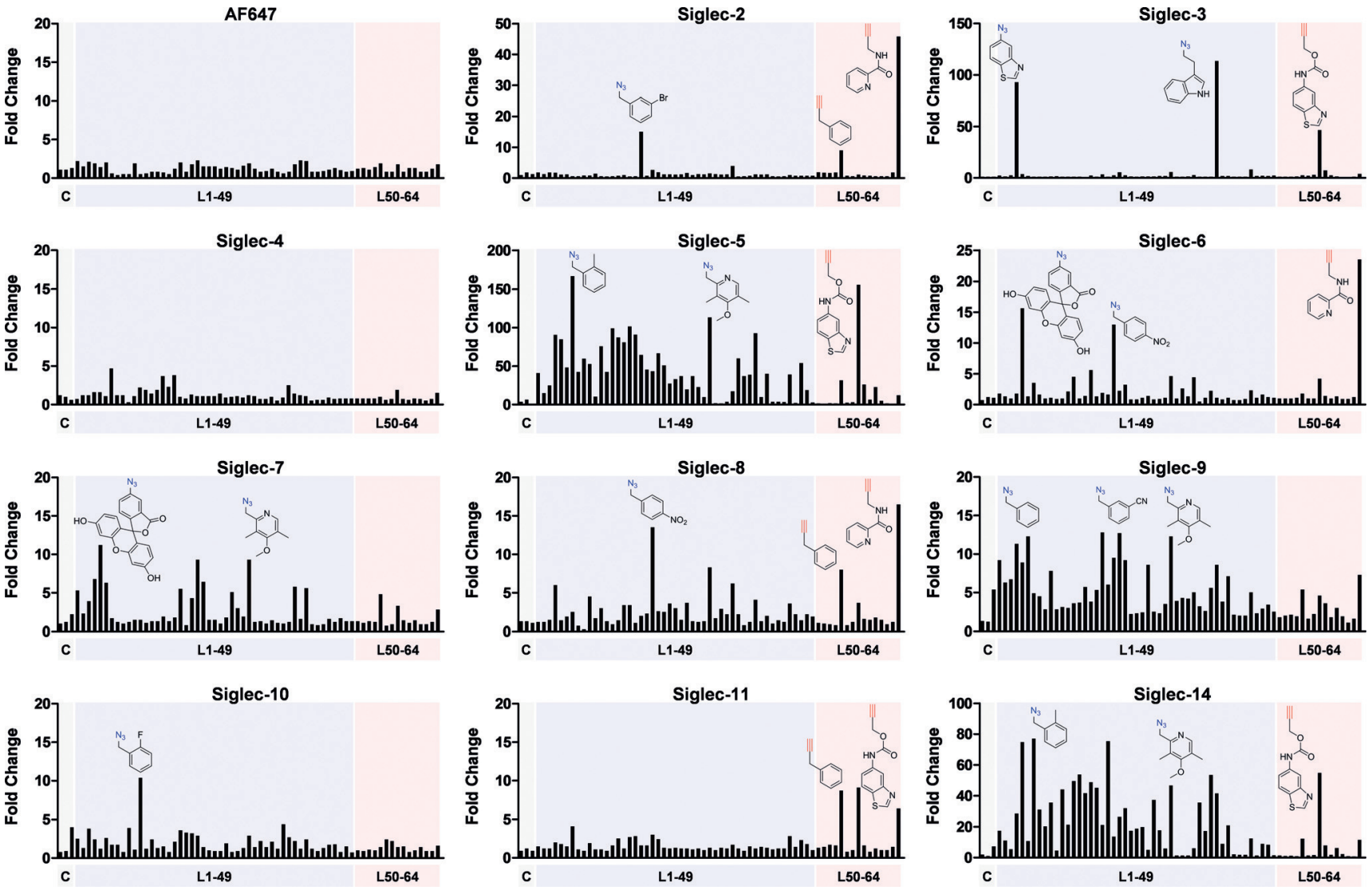

Figure 2. Screening of the sialic acid modified cell library for binding to the human Siglec family.

body) to the modified cells was assessed. To detect binding, the recombinant Siglec-Fc chimeras were precomplexed to Alex Fluor 647 (AF647)-conjugated anti-human-Fc antibodies. The AF647-conjugated anti-human-Fc antibodies alone showed no binding to L1-L64-modified Jurkat cells, but significant changes in binding of the precomplexed Siglec-Fc chimeras to the glycoengineered cell library was observed (Figure 2 and Table S1 in the Supporting Information). The addition of L1-64 to alkyne and azide sialic acid treated cells in the absence of $\mathrm{CuSO}_{4}$ had no effect on Siglec binding, as expected (Figures S6, S7). From the data shown in Figure 2, it is clear that chemical modification of the sialic acids on living cells drastically alters their interaction with Siglecs. Based on the binding patterns, the Siglec family could be roughly divided into two main groups. The first group includes Siglecs$2,-3,-6,-8,-10$, and -11 , which reacted with only a few (1-6) of the sialic acid modifications presented by the cells. The second group, which includes Siglecs-5/-14, -7, and -9, reacted with many (11-49) glycoengineered cells. In contrast, no significant change in the binding of Siglec- 4 to any of the modified cells was found. To confirm applicability across different cells lines, HEK293 kidney cells treated with $\mathrm{Ac}_{5} \mathrm{NeuNPoc}$ were reacted with a selection of azides and screened for Siglec binding. Similar results to those in the Jurkat cell were obtained, which confirmed that the sialic acid modifications specifically enhance Siglec binding (Figure S6). Except for Siglec-4, glycoengineered cells were identified for each Siglec family member tested, including the orphan
Siglecs, Siglecs-6, -8, and -11. Some modifications showed affinity to multiple Siglecs. While these modifications could be useful to target different Siglecs at the same time, selective targeting is required to study or manipulate an individual Siglec family member. Siglecs-5/-14, -7 , and -9 showed similar binding preferences for sialic acids modified with benzyl azides (Figure 2).

To investigate the possibility of selectively targeting individual Siglecs, we modeled the benzyl derivatives into the crystal structure of Siglec-5. ${ }^{[14]}$ This revealed an aspartic acid residue (D130) in the F-G turn close to the sialic acid modification. Sequence alignment of Siglecs-7 and -9 showed that neither of them shares a negatively charged amino acid at this position (Figure S8). We reasoned that positively charged modifications such as L24-L26 could form a salt bridge with D130 and might allow for the selective binding of Siglec-5/-14, which was confirmed by docking experiments (Figure $3 \mathrm{a}$, Figure S11). We therefore synthesized L24-L26, and as predicted, the presence of these positively charged groups on the surface of Jurkat cells strongly enhanced binding to Siglec-5 and its homologue Siglec-14 but not to Siglecs-7 and -9 or any other Siglec family member (Figure $3 \mathrm{~b}$ ). These data demonstrate that, depending on the structure of the V-set domain, clickable molecules can be produced that alter sialic acids selectivity for a particular Siglec member. Moreover, other single (C-2, C-4, C-9) or multiple modifications of the sialic acid backbone could further improve Siglec binding affinity and avidity. ${ }^{[15]}$ 


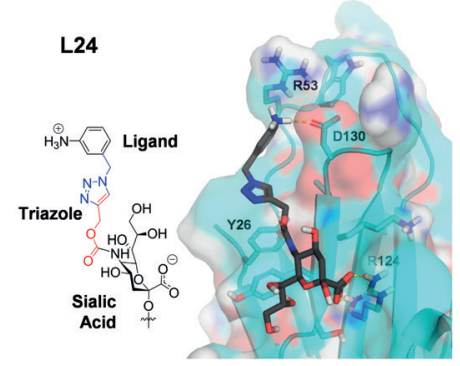

b

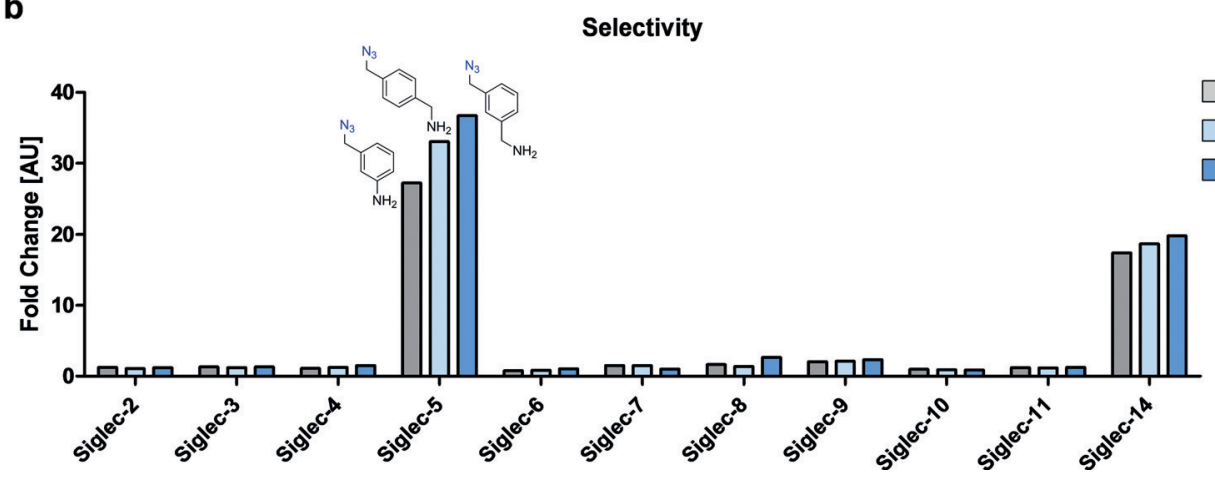

Siglec-5 L25

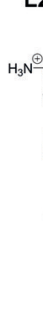

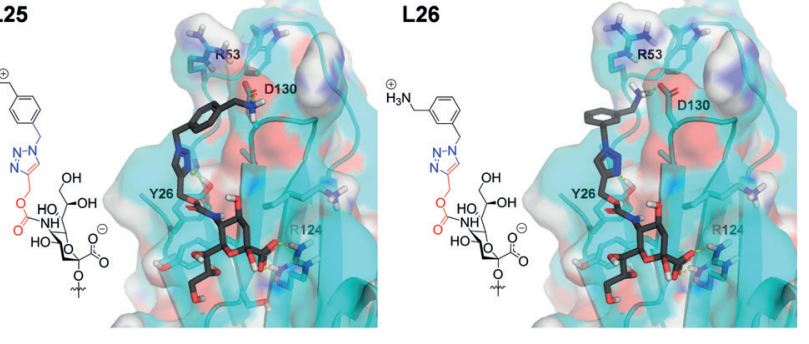

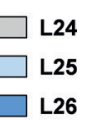

L26

of Siglec-3 in reducing Tolllike receptor and IRF signaling. To further show that the dampening effect of the glycoengineered cells was mediated by Siglec signaling, the reporter cells were pre-treated with the SHP1/-2 inhibitor NSC-87877, which abrogated the inhibiting effects of both the anti-Siglec-3 antibodies and the L4, L39, and L57modifed Jurkat cells (Figure S10). These findings suggest that glycoengineered T-cells can induce immune inhibitory Siglec-3 signaling that counteracts the activation of immune cells through the NF- $\mathrm{KB}$ and IRF pathways. Possibly, the glycoengineered T-cells cross-link Siglec-3, thereby leading to SHP-1/-2 recruit-

Having established the ability to drastically improve Siglec binding affinity and selectivity using bioorthogonal chemistry, we next investigated the functional consequences of such modifications. To this end, we investigated the effect of the interaction between glycoengineered Jurkat T-cells that show high binding to Siglec-3, and Siglec-3-expressing monocytic THP1-DUAL cells. Siglec-3 is normally expressed on myeloid cells, monocytes, myeloid leukemia cells, and microglia cells and contains an intracellular ITIM..$^{[4,7 a]}$ THP1DUAL cells express Siglec-3 but no other Siglec family member (Figure S9) and secrete alkaline phosphatase (AP) in response to immune activation through the NF- $\kappa \mathrm{B}$ pathway, and luciferase (Luc) in response to signaling through the IRF pathway. ${ }^{[16]}$ The two pathways play distinct roles in the activation of the immune system and can be triggered by Tolllike receptor (TLR) ligands. Therefore, this reporter cell model allowed us to study the effect of sialic acid/Siglec-3 interactions on the activation of monocytic cells through the NF- $\kappa \mathrm{B}$ and IRF pathways. ${ }^{[16 \mathrm{~b}, 17]}$ From our glycoengineered cell library (Figure 2), we selected three sialic acid modifications (L4, L39, and L57) that strongly enhance Siglec-3 binding. As a positive control, anti-Siglec-3 antibodies were used that have been shown to cross-link Siglec-3, thereby inducing signaling and SHP-1/-2 recruitment to the ITIM. ${ }^{[18]}$ As a negative control, Jurkat cells modified with L40, which showed no Siglec-3 binding, and unconjugated Jurkat cells were used (Figure 2). Next, the THP1-DUAL cells were activated by TLR ligands LPS and Pam3CSK4, resulting in high production of AP and Luc (Figure 4). Co-culture with unmodified Jurkat cells or Jurkat cells modified with L40 had no significant effect on THP1-DUAL cell activation, whereas cross-linking of Siglec-3 with antibodies reduced AP and Luc secretion. Intriguingly, co-culture with L4, L39, or L57modifed Jurkat cells was as effective as antibody cross-linking ment and suppression of activation through the TLR pathway. Altogether, the described glycoengineering approach can be used to modulate sialic acid/Siglec interactions on a cellular level and may hold therapeutic potential in the context of autoimmune diseases and allergies.

In conclusion, we have developed a glycoengineering approach to readily modify the surface sialic acids of living cells and their Siglec binding. Next to being applied as a type of "glycan array", the glycoengineered cells can be used to study and steer sialic acid/Siglec interactions between living cells. The observed immune suppressive activity of the glycoengineered cells may be applicable to dampen the Finally, the general principle of using bioorthogonal chemistry to edit the glycocalyx and to alter its interaction with lectins may be extended to other classes of sialic acid binding lectins (e.g., selectins, hemagglutinin) or other classes of glycan/lectin interactions (e.g., galectins).

\section{Acknowledgements}

This work was supported by a Radboud University Medical Center PhD Grant awarded to C.B., a VENI grant from the Netherlands Organization for Scientific Research (NWO) awarded to T.J.B., the Netherlands Research School for Chemical Biology (NRSCB) and a KWF grant (KUN20157604) awarded to G.J.A., T.J.B. and C.B.

\section{Conflict of interest}

The authors declare no conflict of interest. immune response in autoimmune diseases and allergies. 


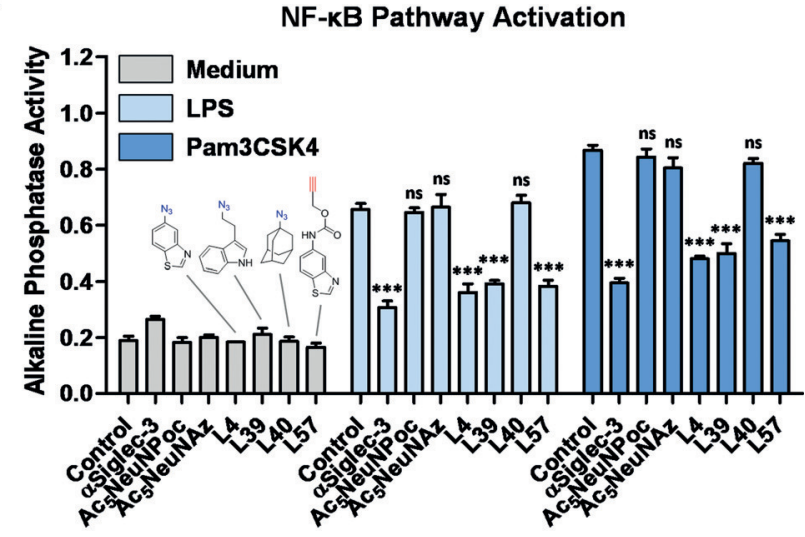

b

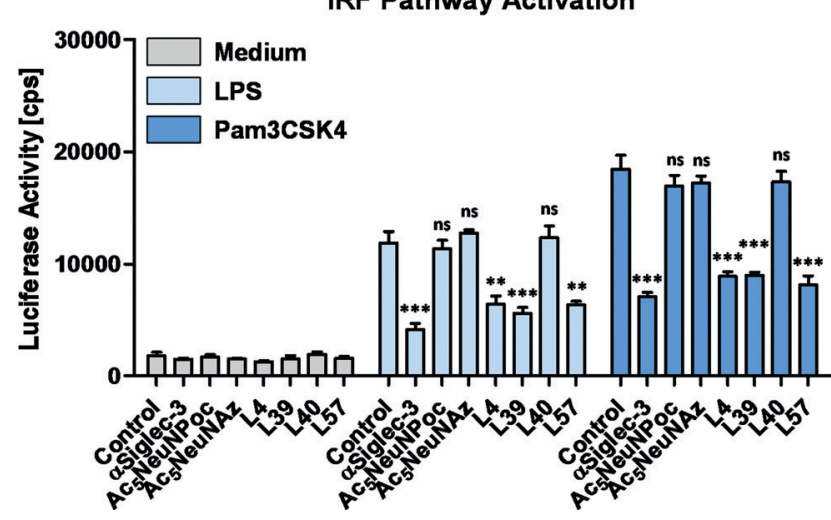

Figure 4. Sialic acid glycoengineered cells that bind Siglec-3 dampen immune activation. a,b) A Siglec-3 cross-linking antibody (positive control) and Jurkat cells expressing L4-, L39-, and L57-modified sialic acids as ligands for Siglec-3 inhibit activation of the NF-KB (a) and IRF (b) pathways by LPS and Pam3CSK4 in Siglec- $3^{+}$monocyte reporter cells as measured by the production of alkaline phosphatase or luciferase, respectively. Unmodified or L40-expressing Jurkat cells that

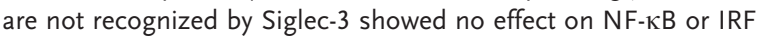
activation.

Keywords: bioorthogonal chemistry · immunology . molecular modeling $\cdot$ sialic acids $\cdot$ Siglecs

How to cite: Angew. Chem. Int. Ed. 2017, 56, 3309-3313 Angew. Chem. 2017, 129, 3357-3361

[1] a) A. Varki, Glycobiology 2011, 21, 1121-1124; b) B. S. Blaum, J. P. Hannan, A. P. Herbert, D. Kavanagh, D. Uhrin, T. Stehle, Nat. Chem. Biol. 2015, 11, 77-82.

[2] a) R. Schauer, Curr. Opin. Struct. Biol. 2009, 19, 507-514; b) A Varki, Nature 2007, 446, 1023-1029; c) P. R. Crocker, J. C. Paulson, A. Varki, Nat. Rev. Immunol. 2007, 7, 255-266.

[3] S. Pillai, I. A. Netravali, A. Cariappa, H. Mattoo, Annu. Rev. Immunol. 2012, 30, 357-392.

[4] M. S. Macauley, P. R. Crocker, J. C. Paulson, Nat. Rev. Immunol. 2014, 14, 653-666.

[5] Y. Ding, Z. Guo, Y. Liu, X. Li, Q. Zhang, X. Xu, Y. Gu, Y. Zhang, D. Zhao, X. Cao, Nat. Immunol. 2016, 17, 1167.

[6] a) I. Surolia, S. P. Pirnie, V. Chellappa, K. N. Taylor, A. Cariappa, J. Moya, H. Liu, D. W. Bell, D. R. Driscoll, S. Diederichs, K.
Haider, I. Netravali, S. Le, R. Elia, E. Dow, A. Lee, J. Freudenberg, P. L. De Jager, Y. Chretien, A. Varki, M. E. MacDonald, T. Gillis, T. W. Behrens, D. Bloch, D. Collier, J. Korzenik, D. K. Podolsky, D. Hafler, M. Murali, B. Sands, J. H. Stone, P. K. Gregersen, S. Pillai, Nature 2010, 466, 243-247; b) T. Toubai, G. Hou, N. Mathewson, C. Liu, Y. Wang, K. OraveczWilson, E. Cummings, C. Rossi, R. Evers, Y. Sun, J. Wu, S. W. Choi, D. Fang, P. Zheng, Y. Liu, P. Reddy, Blood 2014, 123, 3512 3523 ; c) G. Y. Chen, X. Chen, S. King, K. A. Cavassani, J. Cheng, X. Zheng, H. Cao, H. Yu, J. Qu, D. Fang, W. Wu, X. F. Bai, J. Q. Liu, S. A. Woodiga, C. Chen, L. Sun, C. M. Hogaboam, S. L. Kunkel, P. Zheng, Y. Liu, Nat. Biotechnol. 2011, 29, 428-435; d) G. Y. Chen, J. Tang, P. Zheng, Y. Liu, Science 2009, 323, $1722-$ 1725; e) K. Malpass, Nat. Rev. Neurol. 2013, 9, 360; f) C. Büll, M. H. den Brok, G. J. Adema, Biochim. Biophys. Acta Rev. Cancer 2014, 1846, 238-246; g) C. Büll, M. A. Stoel, M. H. den Brok, G. J. Adema, Cancer Res. 2014, 74, 3199-3204.

[7] a) C. Büll, T. Heise, G. J. Adema, T. J. Boltje, Trends Biochem. Sci. 2016, 41, 519-531; b) T. Angata, C. M. Nycholat, M. S. Macauley, Trends Pharmacol. Sci. 2015, 36, 645-660.

[8] a) C. D. Rillahan, E. Schwartz, R. McBride, V. V. Fokin, J. C. Paulson, Angew. Chem. Int. Ed. 2012, 51, 11014-11018; Angew. Chem. 2012, 124, 11176-11180; b) V. V. Rostovtsev, L. G. Green, V. V. Fokin, K. B. Sharpless, Angew. Chem. Int. Ed. 2002, 41, 2596-2599; Angew. Chem. 2002, 114, 2708-2711.

[9] B. Belardi, C. R. Bertozzi, Chem. Biol. 2015, 22, 983-993.

[10] J. E. Hudak, S. M. Canham, C. R. Bertozzi, Nat. Chem. Biol. 2014, 10, 69-75.

[11] a) C. Büll, T. Heise, D. M. Beurskens, M. Riemersma, A. Ashikov, F. P. Rutjes, T. H. van Kuppevelt, D. J. Lefeber, M. H. den Brok, G. J. Adema, T. J. Boltje, ACS Chem. Biol. 2015, 10, $2353-2363 ;$ b) N. J. Agard, C. R. Bertozzi, Acc. Chem. Res. 2009, $42,788-797$.

[12] D. C. Kennedy, C. S. McKay, M. C. Legault, D. C. Danielson, J. A. Blake, A. F. Pegoraro, A. Stolow, Z. Mester, J. P. Pezacki, J. Am. Chem. Soc. 2011, 133, 17993-18001.

[13] a) S. V. Shelke, G. P. Gao, S. Mesch, H. Gathje, S. Kelm, O. Schwardt, B. Ernst, Bioorg. Med. Chem. 2007, 15, 4951-4965; b) S. Kelm, J. Gerlach, R. Brossmer, C. P. Danzer, L. Nitschke, J. Exp. Med. 2002, 195, 1207-1213.

[14] M. A. Zhuravleva, K. Trandem, P. D. Sun, J. Mol. Biol. 2008, 375, $437-447$

[15] a) H. Prescher, A. Schweizer, E. Kuhfeldt, L. Nitschke, R. Brossmer, ACS Chem. Biol. 2014, 9, $1444-1450$; b) H. H. AbduAllah, K. Watanabe, G. C. Completo, M. Sadagopan, K. Hayashizaki, C. Takaku, T. Tamanaka, H. Takematsu, Y. Kozutsumi, J. C. Paulson, T. Tsubata, H. Ando, H. Ishida, M. Kiso, Bioorg. Med. Chem. 2011, 19, 1966-1971; c) S. Kelm, P. Madge, T. Islam, R. Bennett, H. Koliwer-Brandl, M. Waespy, M. von Itzstein, T. Haselhorst, Angew. Chem. Int. Ed. 2013, 52, 3616-3620; Angew. Chem. 2013, 125, 3704-3708.

[16] a) B. Dey, R. J. Dey, L. S. Cheung, S. Pokkali, H. Guo, J. H. Lee, W. R. Bishai, Nat. Med. 2015, 21, 401-406; b) K. Honda, T. Taniguchi, Nat. Rev. Immunol. 2006, 6, 644-658.

[17] a) W. Chen, C. Han, B. Xie, X. Hu, Q. Yu, L. Shi, Q. Wang, D. Li, J. Wang, P. Zheng, Y. Liu, X. Cao, Cell 2013, 152, 467-478; b) G. Y. Chen, N. K. Brown, W. Wu, Z. Khedri, H. Yu, X. Chen, D. van de Vlekkert, A. D'Azzo, P. Zheng, Y. Liu, elife 2014, 3, e04066.

[18] V. C. Taylor, C. D. Buckley, M. Douglas, A. J. Cody, D. L. Simmons, S. D. Freeman, J. Biol. Chem. 1999, 274, 11505-11512.

Manuscript received: December 15, 2016

Final Article published: February 14, 2017 